\title{
Design and Evaluation of a Compact Antenna for Implant-to-Air UWB Communication
}

\author{
Tharaka Dissanayake, Member, IEEE, Mehmet R. Yuce, Member, IEEE, and Chee Ho
}

\begin{abstract}
A compact, printed, capsule-shaped ultrawideband (UWB) antenna for biomedical implants is presented. The 10-dB return loss bandwidth of the proposed antenna is $3.5-4.5 \mathrm{GHz}$, which covers the recently proposed UWB for biomedical applications. The antenna matching has been optimized using CST Microwave Studio and verified by measurements. An optimized feed mechanism to reduce guided wavelength, as well as integration with other electronics, has been considered in the antenna design. Radiation performance has been evaluated on an impulse-based UWB transmitter/receiver testbed to show that the antenna is usable in biomedical applications, such as capsule endoscopes. The antenna has been tested both in free space and with tissue loading.
\end{abstract}

Index Terms-Biomedical communication, broadband communication, printed antennas, ultrawideband (UWB) antennas.

\section{INTRODUCTION}

C APSULE endoscope is an alternative to a fiber-based endoscope used in diagnosing diseases related to the gastrointestinal tract. It removes the inconvenience and pain suffered by the patients when wired endoscopes are used. A capsule endoscope can also reach areas such as the small intestine and deliver real-time images wirelessly to an external console. In addition to image data, navigation and control of a sophisticated capsule endoscope should be delivered over a high-capacity wireless link. For high data rate and short-range applications of this kind, ultrawideband (UWB) communication is an ideal physical-layer solution. Moreover, impulse-based UWB transmitters are simple and can be built compactly even with offthe-shelf components, ideal for size-restricted biomedical applications. UWB standards leave almost $7 \mathrm{GHz}$ of frequency bandwidth license-free for low-power operation. Recently, a working group for Body Area Networks (BAN) known as IEEE 802.15.6 has been formed to develop a communication standard optimized for low-power devices operating near, on, or inside the human body. IEEE 802.15.4a, a UWB-based standard approved in March 2007, is one of the potential candidates for BAN applications, such as the capsule endoscope [7]. The frequency band

Manuscript received October 23, 2008; revised December 03, 2008. First published January 20, 2009; current version published April 22, 2009.

The authors are with the are with School of Electrical Engineering and Computer Science, University of Newcastle, Callaghan, NSW 2308, Australia (e-mail: tharaka.dissanayake@newcastle.edu.au).

Color versions of one or more of the figures in this letter are available online at http://ieeexplore.ieee.org.

Digital Object Identifier 10.1109/LAWP.2009.2013370
3.5-4.5 GHz, selected for our UWB development, avoids narrowband systems operating in the industry, scientific, and medical (ISM) bands. Furthermore, beyond $5 \mathrm{GHz}$, the tissue imposes strong attenuation to electromagnetic waves [2]. The antennas and applications that have been previously reported for endoscope applications operate in lower frequency bands [3] for this reason. Therefore, to obtain the full use of high data rate capabilities of UWB while minimizing through-body losses, it is best to select the lower end of the UWB band. The printed antenna presented in this letter is shaped to be used in a capsule and demonstrates good matching in the frequency band of $3.5-4.5 \mathrm{GHz}$. The radiation performance has been evaluated experimentally using an impulse-based UWB transmitter/receiver testbed to show that it is suitable for endoscope-type implanted applications.

\section{Antenna Design}

\section{A. Configuration}

The proposed antenna is printed on a 0.5 -mm-thick RO4003 dielectric substrate with dielectric constant of 3.38. The substrate takes the shape of a capsule. It can fit inside a size 13 capsule [4], which may easily support a UWB transmitter built out of discrete components, a sensor, and a small battery. Overall length and width of the antenna is 28.7 and $14 \mathrm{~mm}$, respectively. It is essentially a planar dipole, which has been optimized using CST Microwave Studio (commercial electromagnetic simulation software) and printed on one side of the substrate together with a grounded coplanar waveguide (CPW) feed as shown in Fig. 1. Grounded CPW has characteristic impedance of $50 \Omega$, and the ground plane on the opposite side of the substrate is intended to support other electronics. This avoids performance degradation upon integration with the transmitter, sensors, batteries and connectors. In our prototypes, we used a panel-mount SMA connector instead to connect the antenna to the testbed (see Fig. 1). Note that the flange of the connector acts as a ground plane to the CPW. The circular pad in one end of the grounded CPW facilitates broadband coaxial-to-CPW transition [5]. The center conductor of the connector is soldered to the circular pad. When the SMA connector is replaced by the on-board transmitter, a differential feed between the pad and the ground can be employed.

The feed line has an effective dielectric constant of 2.62 at $3.5 \mathrm{GHz}$ (lower end of the matched band). Therefore, the guided wavelength at the same frequency is approximately $53 \mathrm{~mm}$. The overall antenna length is $28.7 \mathrm{~mm}$, which is close to half the guided wavelength, typical for a dipole. Note that this guided wavelength is less than $62 \mathrm{~mm}$, which is the guided wavelength 


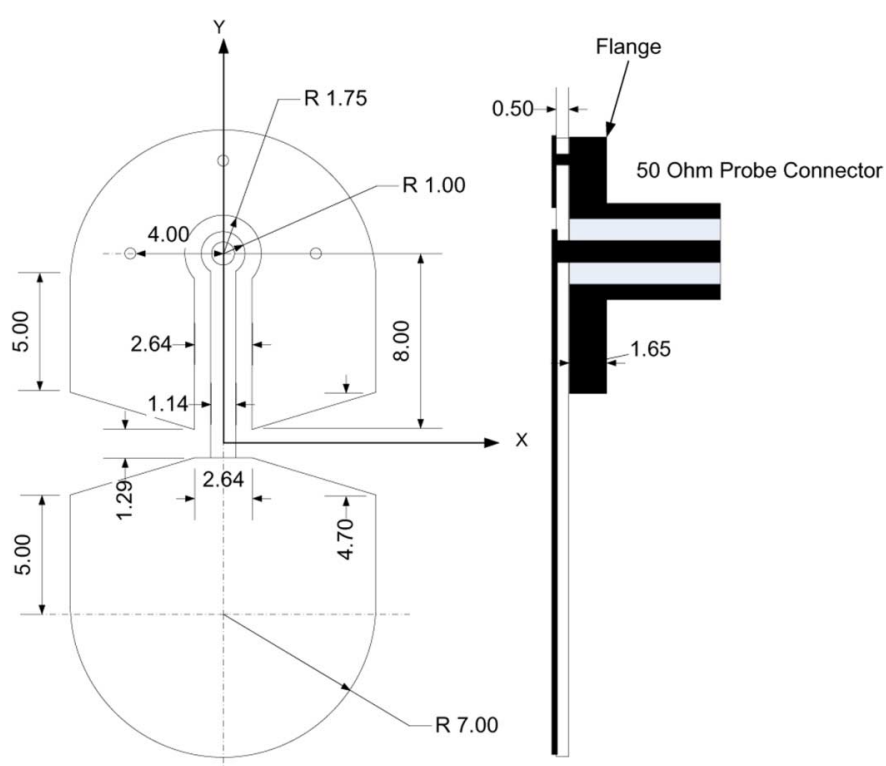

Fig. 1. Patch dimensions (left) and cross section (right) of the proposed capsule-shaped UWB antenna.

of a CPW without ground at $3.5 \mathrm{GHz}$. This is because the grounded $\mathrm{CPW}$ has most of its electric fields confined to the substrate compared to a CPW with nearly half of the electric field lines occupying free space. The additional ground plane supporting the feed contributes to the miniaturization of the antenna by reducing the guided wavelength by approximately $10 \mathrm{~mm}$. As a result, the largest dimension of the proposed antenna is only 0.3 times the free space wavelength at $3.5 \mathrm{GHz}$, $40 \%$ less compared to half-wavelength.

Three symmetrically placed vias ensure electrical connection between the patch on one side of the substrate and the flange of the connector on the other side. The radius of each via is $0.75 \mathrm{~mm}$. Although the number of vias does not make a significant difference, parametric studies have shown that the distance to the vias from the center of the coaxial feed affects the input impedance of the antenna. Note that the patch, flange, and each via form transmission lines, which may act as shorted transmission line resonators. At certain lengths, this resonant frequency can be between 3.5 and $4.5 \mathrm{GHz}$, creating an in-band notch, which is not desirable. Thus, we have selected $4 \mathrm{~mm}$ as the optimum distance after a parametric study, which has also shown that larger via diameters create good connectivity.

\section{B. Impedance Matching}

Two antenna prototypes have been fabricated using conventional printed circuit board (PCB) design techniques. Reflection coefficients of both antennas have been measured using the E5071B vector network analyzer from Agilent. Measured results and simulated S11 values from CST Microwave Studio are shown in Fig. 2. There is a good agreement between measured and theoretical $S 11$ results. Antennas have greater than 10-dB return loss from 3.4-4.6 GHz. Frequently used PCB etching and soldering methods have been used for prototyping. Still, the matching performance of both antenna prototypes is

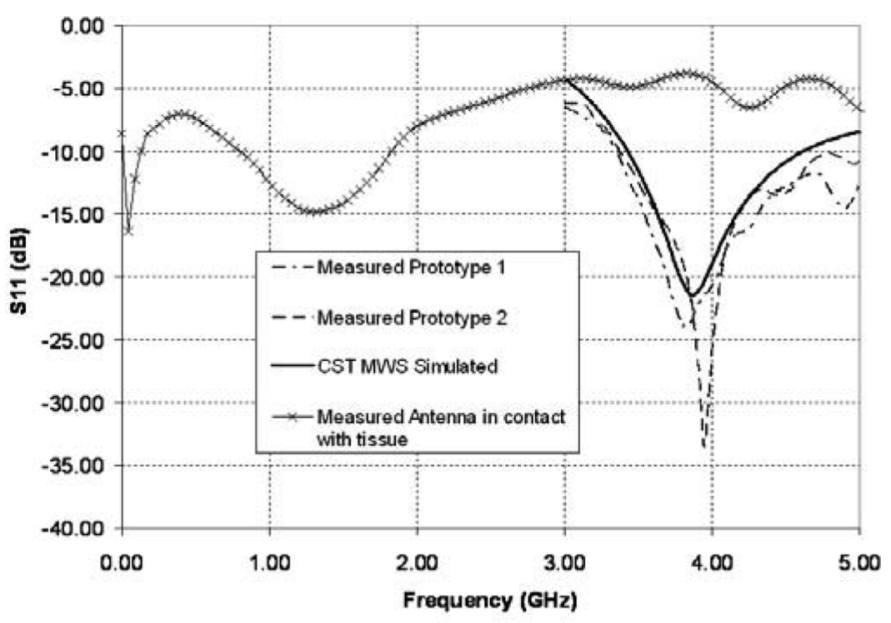

Fig. 2. Measured and simulated reflection coefficients of the UWB antenna prototypes.

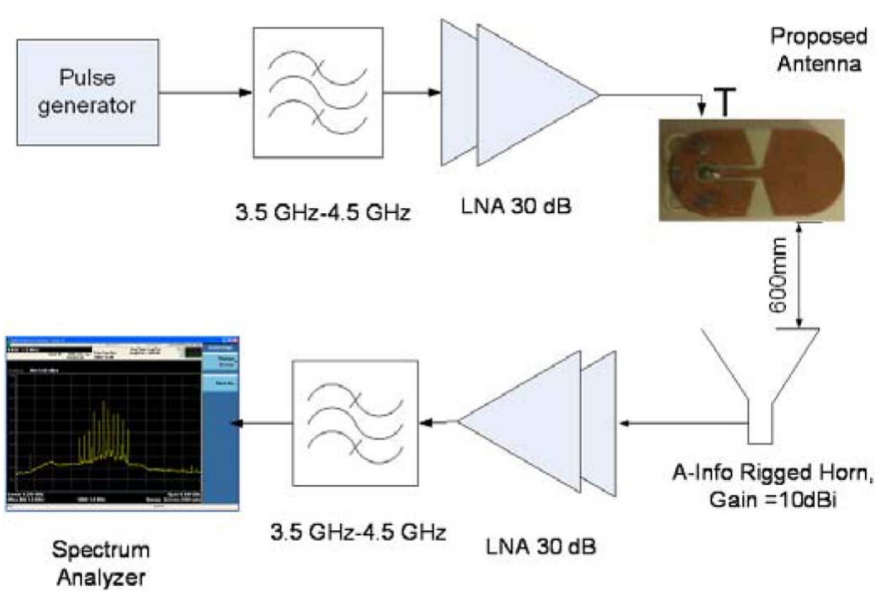

Fig. 3. Experiment setup with broadband horn antenna at the receiver.

promising. A design robust against tolerances can be successfully integrated to a miniature biomedical device.

\section{RADIATION PERFORMANCE}

Simulations suggests that the proposed antenna, with the SMA connector resembling transmitter and sensor electronics, has radiation patterns (discussed later) similar to that of a dipole antenna. Theoretical gain at $4 \mathrm{GHz}$ is $2.23 \mathrm{~dB}$. More important here is to demonstrate that this antenna is capable of supporting an impulse-based UWB system, which will be integrated with the antenna for an application similar to the capsule endoscope. Therefore, the antenna has been tested on an impulse-based UWB transmitter/receiver testbed that has been developed in-house (shown in Fig. 3). The UWB source generates a stream of 660-ps pulses with 130-ps rise time, repeated at a $200-\mathrm{MHz}$ frequency. The spectrum of thus-generated pulses extends over an unlimited frequency band [6]. A band-pass filter (BPF) centered at $4 \mathrm{GHz}$ with $1-\mathrm{GHz}$ bandwidth is used to limit the signal power within the desired band as shown in Fig. 4 (resolution bandwidth is $1 \mathrm{MHz}$ ). Note that Fig. 4 shows the measured spectrum at point $\mathrm{T}$ in Fig. 3, prior to amplifiers and band-pass filters in the receiver. The filtered pulses are fed into the proposed UWB antenna, which also has its center 


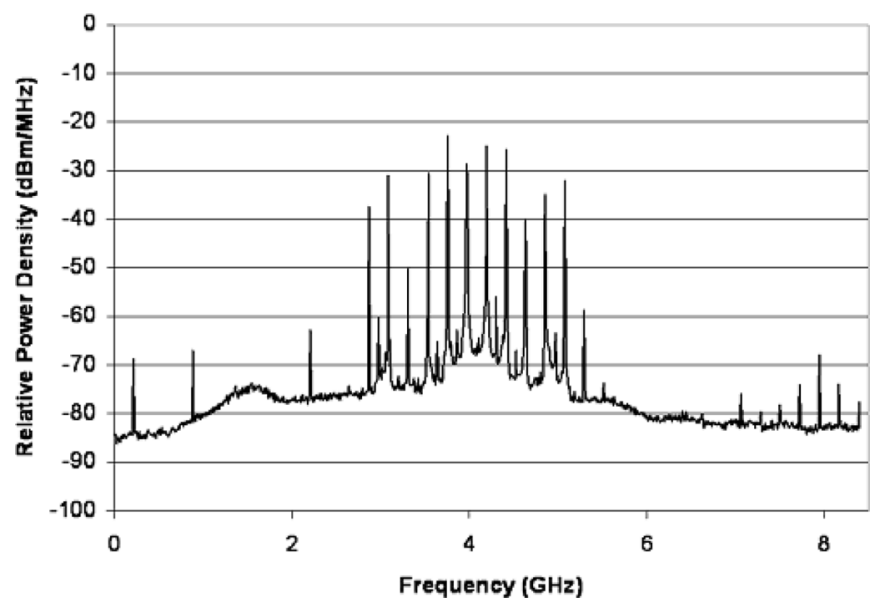

Fig. 4. Band-limited transmitted signal spectrum at the terminals of the UWB antenna at point $\mathrm{T}$.

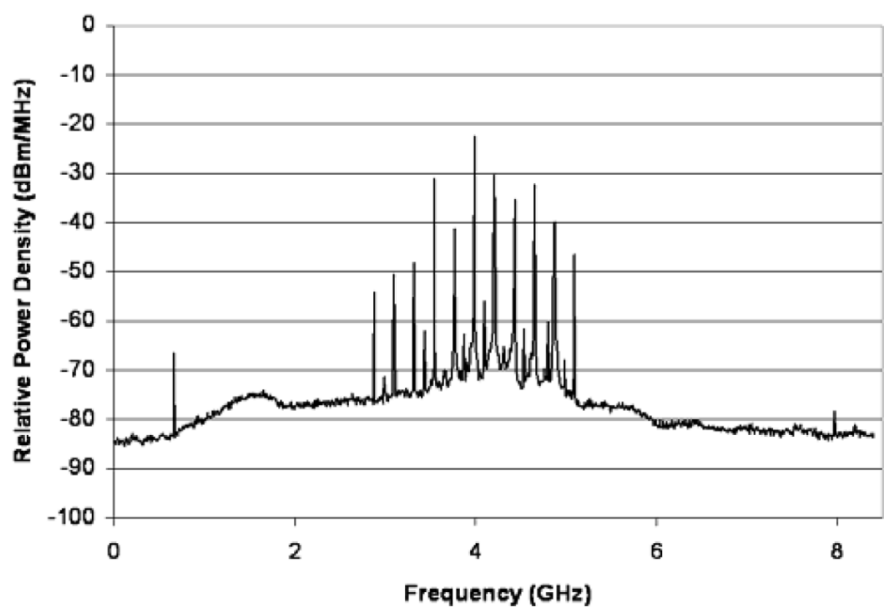

Fig. 5. Measured average received power spectral density through free space.

frequency around $4 \mathrm{GHz}$. The antenna shapes the UWB pulses further, limiting out-of-band frequency components.

\section{A. Free-Space Experiment}

The UWB signal was successfully detected by a broadband horn antenna by A-Info, which was $600 \mathrm{~mm}$ away in the boresight of the transmitter antenna with direct line-of-sight within the laboratory environment. The received signal is passed through a BPF, whose center frequency is $4 \mathrm{GHz}$, to eliminate possible interference from the frequencies of wireless local area network (WLAN) standards (for example, 2.4 and $5 \mathrm{GHz}$ ). The signal is then amplified by a couple of low noise amplifiers (LNA). The measured average signal spectrum at the output of the receiver BPF is shown in Fig. 5. Most of the out-of-band frequency components from the UWB pulse generator have been removed by the filters and the capsule-shaped antenna itself. Moreover, in-band (3.5-4.5 GHz) components of the spectrum are very similar to those shown in Fig. 4, the transmitted spectrum at the antenna input $\mathrm{T}$. In other words, propagation loss through antennas and free space has been compensated by the LNAs in the receive chain. The UWB signal is thus recoverable using an energy detector at the output of the BPF. Free-space path loss is about $40 \mathrm{~dB}$. The A-Info antenna has 10-dBi gain

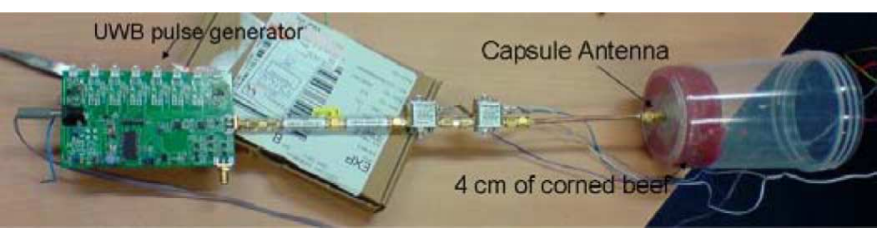

Fig. 6. Antenna loaded with meat connected to the pulse generator and the RF chain.

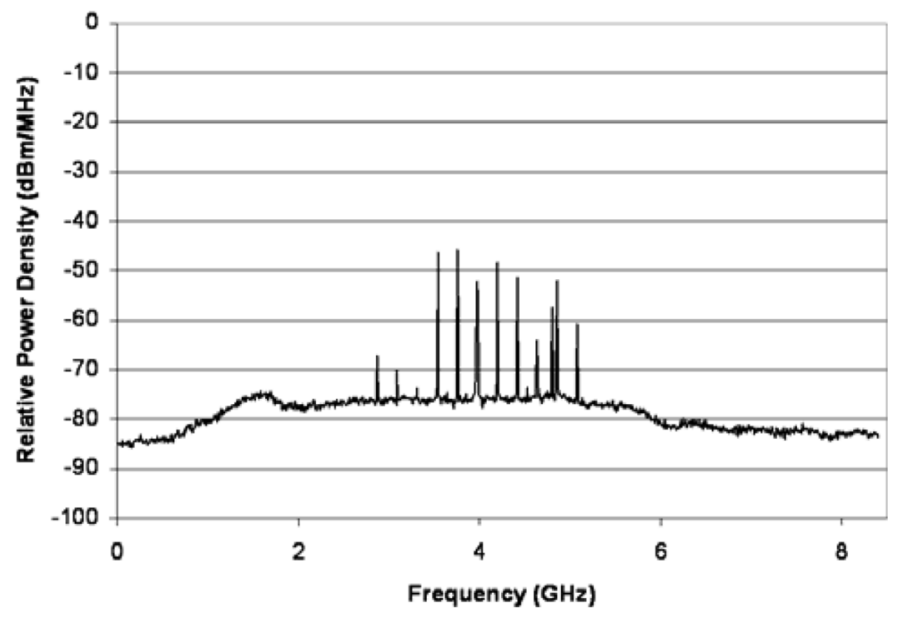

Fig. 7. Measured average received-power spectral density through $40 \mathrm{~mm}$ of meat and free space.

at $4 \mathrm{GHz}$. Therefore, the capsule-shaped antenna gain can be estimated to be $2-5 \mathrm{dBi}$.

\section{B. Through-Meat Experiment}

The capsule-shaped antenna has been loaded with a cylinder of corned beef, having thickness of $4 \mathrm{~cm}$ and diameter of $75 \mathrm{~mm}$, inside a plastic container as shown in Fig. 6. The container does not have any effect on the measurements at the frequencies of concern.

The antenna was covered with a thin coating of clear rubber from Chemsearch ${ }^{\mathrm{TM}}$ to protect it from moisture, meat, and blood. Corned beef has a high salt content, and with the antenna firmly flushing against the tissue, it resembles a worst-case scenario in terms of efficiency and S11 changes. The orientations of both antennas are similar to those in the previous experiment where the line-of-sight coupling is in the boresight. Distance between the surface of the meat sample and the horn antenna also remained at $600 \mathrm{~mm}$.

Fig. 7 shows the measured spectrum after propagation through the meat sample and then the free space. The information is still sufficient to recover the pulse train using an energy detector. Comparing Figs. 5 and 7, it can be noted that there is about a $25-\mathrm{dB}$ signal attenuation due to the introduction of meat. The major effect of the antenna in contact with the tissue as an implant is the shift of $10-\mathrm{dB}$ return loss bandwidth to lower frequencies. As shown in Fig. 2, the matched bandwidth has moved to $800 \mathrm{MHz}-1.8 \mathrm{GHz}$. Effectively, this is antenna miniaturization, which is consistent with previous studies in $\mathrm{GHz}$ frequencies [1]. Overall, the channel demonstrates frequency-selective fading characteristics and, hence, dispersion. This is due to near-field and far-field attenuation in the tissue 


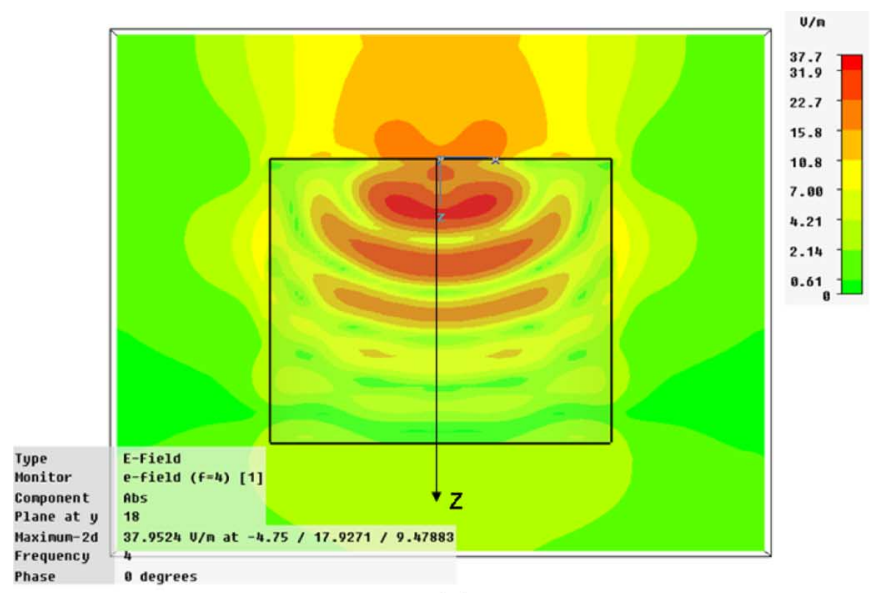

(a)

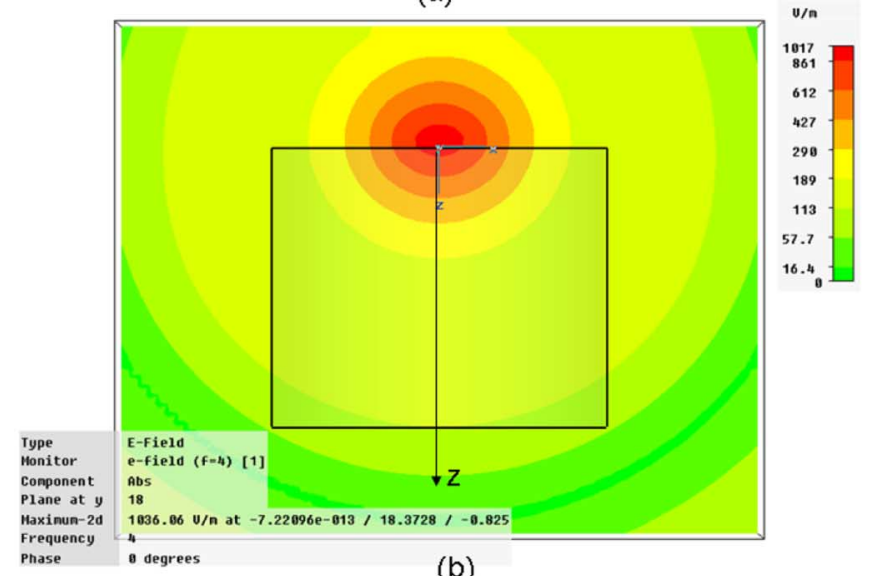

(b)

Fig. 8. Absolute electric field distribution in the azimuth plane of the capsuleshaped UWB antenna. (a) Loaded with tissue simulating material; (b) in free space (outline of the tissue cylinder is shown for comparison).

[8], the change of far-field radiation pattern of the transmitting antenna, and, thereby, the scattering.

The difference of absolute electric field distribution in the azimuth plane at $4 \mathrm{GHz}$ between the loaded and unloaded UWB antennas is shown in Fig. 8(a) and (b), respectively. In this CST MWS simulation, the antenna is flushed against a cylinder of tissue simulating material $\left(\varepsilon_{r}=45, \sigma=2 S / m\right)$ [9]. Diameter of the cylinder is $60 \mathrm{~mm}$, and the height is $40 \mathrm{~mm}$. Its axis is along the z-axis.

There is a significant variation of near- and far-fields as a result of loading the antenna with a tissue-simulating material. The amplitude of the electric field is reduced by several orders of magnitude. The strength of the far-field toward the z-direction, where the horn antenna is receiving the signal, has also been reduced. In Fig. 8(a), highest field strength is observed inside the tissue sample, well in contrast with the omnidirectional distribution shown in Fig. 8(b). This difference in field patterns causes the scattering properties of the channel to change, resulting in frequency-selective (dispersive) characteristics.

\section{CONCLUSION}

The proposed capsule-shaped UWB antenna has shown good performance in the frequency band of 3.5-4.5 GHz. It has also shown its ability to form a 0.6-m UWB link across the laboratory both in free space and when loaded with meat emulating an implant. With all the losses due to mismatch and tissue conductivity, we are convinced that a high-gain antenna at the receiver can ensure receiving of a band-limited UWB signal emitted by the proposed low-gain antenna through tissue at a fair distance. The transmitter peak power level is approximately in the range of $-20 \mathrm{dBm}$ for our experiments. However, considering the strong attenuation through body tissue, the power level can be adjusted to $0 \mathrm{dBm}$ without violating power levels of regulation. This is further supported by the low gain of the proposed antenna. The A-Info horn antenna used in the experiment has a wide bandwidth and high gain. We envision future UWB radio links supporting the proposed bandwidth and having a low-gain, compact, capsule-shaped antenna at the implanted transmitter and a high-gain directional antenna or an array of antennas at the receiver.

\section{REFERENCES}

[1] S. Soora, K. Gosalia, M. S. Humayan, and G. Lazzi, "A comparison of two and three dimensional dipole antennas for an implantable retinal prosthesis," IEEE Trans. Antennas Propag., vol. 56, no. 3, pp. 622-629, Mar. 2008.

[2] J. Wang and D. Su, "Design of an ultra wideband system for in-body wireless communications," in Proc. 2006 4th Asia-Pacific Conf. Environmental Electromagn., Dalian, China, 2006, pp. 565-568.

[3] S. i. Kwak, K. Chang, and Y. J. Yoon, "Ultra-wide band spiral shaped small antenna for the biomedical telemetry," in Proc. Asia Pacific Microw. Conf., 2005, vol. 1.

[4] Torpac Inc., "Capsule size chart," Fairfield, NJ, 2000.

[5] T. Kamei, Y. Utsumi, N. Q. Dinh, and N. Thanh, "Wide-Band coaxial-to-coplanar transition," IEICE Trans. Electron., vol. E90-C, no. 10, pp. 2030-2036, 2007.

[6] M. R. Yuce, W. Liu, M. S. Chae, and J. S. Kim, "A wideband telemetry unit for multi-channel neural recording systems," in Proc. IEEE Int. Conf. Ultra-Wideband (ICUWB), Sep. 2007, pp. 612-617.

[7] IEEE, "IEEE 802.15 WPANTM Task Group 6 (TG6) body area networks," May 2008 [Online]. Available: http://www.ieee802.org/15/ pub/TG6.html

[8] S. K. S. Gupta, S. Lalwani, Y. Prakash, E. Elsharawy, and L. Schwiebert, "Towards a propagation model for wireless biomedical applications," in Proc. IEEE Int. Conf. Commun., May 2003, vol. 3, pp. 1993-1997.

[9] M. A. Stuchly, A. Krazewski, S. S. Stuchly, and A. M. Smith, "Dielectric properties of animal tissues in vivo at radio and microwave frequency: Comparison between species," Physics in Medicine and Biology, vol. 27, no. 7, pp. 927-936, 1982. 